\title{
INFORMAÇÃO SOBRE SECA E SAÚDE: SUBSÍDIO PARA AÇÕES PREVENTIVAS
}

\author{
Aderita Sena \\ aderitasena@gmail.com \\ Christovam Barcellos \\ $\underline{\text { xris@ fiocruz.br }}$
}

\begin{abstract}
Resumo
Este estudo aprofunda o conhecimento sobre o processo natural da seca, um evento climático extremo e complexo que tende a se tornar um desastre social e um problema de saúde pública, quando não reconhecido pelo setor saúde. Os riscos sobre os determinantes ambientais, sociais e econômicos que são acentuados pela seca e os impactos sobre o bem-estar e a saúde humana são descritos com o propósito de fortalecer a organização e disseminação de informação, bem como o desenvolvimento de ações para o setor saúde. Ações intersetoriais são recomendadas para o governo e a sociedade atuarem conjuntamente em medidas de prevenção e redução de risco associados à seca, adaptação e resiliência para fortalecer a infraestrutura governamental e o desenvolvimento humano na região. O planejamento de ações deve levar em consideração os dados sobre seca e saúde disponíveis, as experiências passadas e o saber e a cultura das populações afetadas.
\end{abstract}

Palavras-chave: Saúde. Seca. Semiárido Brasileiro. Conhecimento. Informação em saúde. Tomada de decisão. Redução de risco. Adaptação. Resiliência.

\section{DRY AND HEALTH INFORMATION: PREVENTIVE ACTION GRANT}

\begin{abstract}
This study deepens knowledge about the natural process of drought, an extreme and complex climate event that tends to become a social disaster and a public health problem when not recognized by the health sector. The risks to the environmental, social and economic determinants that are accentuated by drought and the impacts on human health and well-being are described for the purpose of strengthening the organization and dissemination of information, as well as the development of actions for the sector. Cheers. Intersectoral actions are recommended for government and society to work together on drought prevention, risk reduction, adaptation and resilience measures to strengthen government infrastructure and human development in the region. Action planning should take into account available drought and health data, past experiences, and the knowledge and culture of affected populations.
\end{abstract}

Keywords: Health. Drought. Brazilian semiarid. Knowledge. Health information. Decision making. Risk reduction. Adaptation. Resilience.

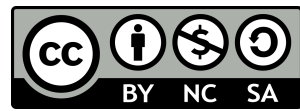




\section{INTRODUÇÃO}

Evidências científicas mostram que a Terra está sofrendo impactos em seus sistemas natural e biofísico e em todas as dimensões de sustentabilidade (WHITMEE et al., 2015). Um estudo realizado em 2005 pela Avaliação Ecossistêmica do Milênio (em sua sigla em inglês MEA, Millenium Ecosystem Assessment) havia alertado que os ecossistemas do planeta estavam sendo utilizados de forma insustentável, com $60 \%$ dos seus serviços já degradados. Segundo esse estudo, a exploração sem limites dos recursos provenientes dos ecossistemas ameaçaria a sustentabilidade das futuras gerações (CORVALAN et al., 2005). A partir desse estudo, outras avaliações realizadas pelo Global Environment Outlook (UNEP, 2012) e pelo Painel Intergovernamental de Mudanças Climáticas (IPCC, 2014) reportam que a saúde humana global tende a ser seriamente ameaçada devido a alteração acelerada dos sistemas naturais da Terra.

As atividades humanas têm funcionado como forças motrizes para alterações no sistema climático, influenciando sobre os sistemas naturais e humanos (McMICHAEL et al., 2008; BARCELLOS et al., 2009). Os danos a esses sistemas estão ameaçando os ganhos conquistados recentemente e pondo em ameaça a vida das futuras gerações. Além disso, os riscos advindos das mudanças ambientais globais vêm se agravando nas últimas décadas. Essas mudanças são decorrentes de processos como mudanças climáticas, acidificação dos oceanos, degradação do solo, perda de biodiversidade, destruição de ecossistemas, exploração insustentável de recursos naturais, uso e ocupação do solo de forma inadequada, desmatamento e processo de desertificação (PATZ et al., 2012; WHITMEE et al., 2015).

Eventos climáticos extremos, como tempestades, inundações, ciclones, secas, ondas de calor e de frio estão ocorrendo de forma mais intensa e com mais frequência ao redor do mundo (IPCC, 2012; 2014). Em muitos países a alteração nas médias anuais de precipitação estão influenciando nos sistemas hidrológicos, ampliando os eventos de seca e inundações, com projeção para piorarem em um futuro próximo (IPCC, 2014; MARENGO et al., 2017). As secas são eventos climáticos complexos. Sua evolução lenta e silenciosa se tornam potenciais riscos para os sistemas naturais e humanos (CARVALHO, 2012; HAGENLOCHER et al., 2019). Seus impactos são múltiplos e severos, podendo causar danos diretos e indiretos sobre os determinantes ambientais, econômicos, sociais (MAGALHÃES, 2016; HAGENLOCHER et al., 2019; MEZA et al., 2019) e sobre a saúde (CDC, 2010; STANKE et al., 2013; SENA et al., 2014). Esses riscos põem em ameaça o suprimento de água potável, os sistemas agrícolas e a segurança alimentar, e contribui para a pobreza e desigualdades, alterando as condições de vida e de saúde das populações (MYERS; PATZ, 2009; LIMA et al., 2011; PATZ et al., 2012; OPAS, 2015).

Junto a esses múltiplos riscos a evidência mostra que $40 \%$ das terras do nosso planeta são consideradas áridas e secas, que podem se expandir ao longo das próximas décadas (UNCCD, 2011; 2016). A preocupação aumenta quando evidências apontam que existem poucas terras disponíveis para 
produção agrícola no mundo (FAO, 2013) e que se projeta até 2050 um aumento possivelmente de até $50 \%$ de demanda de água (incluindo todos os seus usos) (DAMANIA et al., 2017) e de 70\% de alimentos (FAO; WWC, 2015). Dentro de um futuro próximo, será possível alimentar uma população de mais de 7 bilhões de pessoas com projeção para mais de 9 bilhões em 2050? Alguns estudos apontam que insegurança alimentar e nutricional tendem a persistir e ameaçar muitas partes do mundo, principalmente regiões e populações mais pobres e remotas (FAO; WWC, 2015).

Todos esses problemas se constituem em ameaças para um desenvolvimento humano sustentável. A Figura 1 apresenta os efeitos da pressão humana sobre os sistemas naturais da Terra e as consequências sobre o ambiente e a sociedade com os possíveis impactos sobre a saúde humana. Os impactos sobre o ambiente e a sociedade pode ser decorrente de efeitos primários: o caso de ocorrência de desastres e estresse por calor atribuídos aos efeitos das mudanças climáticas, exposição a poluentes; secundários: os fatores mediados pela alteração nos sistemas naturais, como exemplo os impactos sobre água, alimentos, ar, solo, fibras, plantas medicinais etc. e terciários: os impactos associados a ruptura de estruturas sociais e econômicas da sociedade, os quais podem consistir em longa duração (BUTLER et al., 2014).

Figura 1 - Impactos das mudanças ambientais globais sobre os recursos naturais essenciais ao sustento da vida e sobre a saúde humana

Pressão humana sobre as
mudanças ambientais
Mudanças climáticas
Desmatamento
Degradação da terra
Mudança de cobertura da terra
Desertificação
Diminuição da camada de ozônio
Perda e danos de zonas úmidas
Esgotamento e
contaminação de água doce
Urbanização e seus efeitos
sobre o ambiente
Danos nos recifes costeiros
Danos nos ecossistemas
Perda de biodiversidade

\begin{tabular}{|c|c|c|}
\hline $\begin{array}{c}\text { Efeitos sobre } \\
\text { o ambiente e a sociedade }\end{array}$ & & $\begin{array}{l}\text { Efeitos sobre a } \\
\text { saúde humana }\end{array}$ \\
\hline Primários (Diretos) & \multirow{8}{*}{$-\rightarrow$} & - Desnutrição \\
\hline $\begin{array}{l}\text { Desastres (inundaçōes, secas, } \\
\text { ondas de calor, deslizamento de terras, } \\
\text { tempestades, ciclones) }\end{array}$ & & $\begin{array}{l}\text { - Deficiência de } \\
\text { nutrientes }\end{array}$ \\
\hline Escassez de água & & \\
\hline Exposição à radiação ultravioleta & & - Afogamento/ \\
\hline $\begin{array}{l}\text { Exposição a poluentes e alergênicos (ar, } \\
\text { solo, água e alimentos) }\end{array}$ & & - Doenças \\
\hline $\begin{array}{l}\text { Secundários (por meio de alteração nos } \\
\text { ecossistemas) }\end{array}$ & & cardiovasculares \\
\hline Redução na produção de alimentos & & •Doenças \\
\hline Alteração no risco de doenças infecciosas & & respiratórias \\
\hline $\begin{array}{l}\text { Esgotamento de medicamentos naturais } \\
\text { (plantas medicinais) e de outros recursos } \\
\text { naturais (água, fibras, nutrientes) }\end{array}$ & \multirow[t]{4}{*}{$--\Rightarrow$} & - Doenças infecciosas \\
\hline Aumento de poluentes e alergênicos & & $\begin{array}{l}\text { - Doenças infecciosas } \\
\text { transmitidas por }\end{array}$ \\
\hline $\begin{array}{l}\text { Terciários (Indiretos por meio de ruptura } \\
\text { social e econômica) }\end{array}$ & & vetores \\
\hline Perda de subsistência & & - Doenças mentais \\
\hline $\begin{array}{l}\text { Deslocamento da população (aumento } \\
\text { de habitação em favelas) }\end{array}$ & \multirow[t]{3}{*}{$-\rightarrow$} & - Aumento de \\
\hline $\begin{array}{l}\text { Empobrecimento da economia, cultura e } \\
\text { desenvolvimento humano }\end{array}$ & & doenças crônicas \\
\hline Conflitos por água, terra e alimentos & & - Câncer \\
\hline
\end{tabular}

Fonte: Adaptado de Corvalan et al. (2005), Butler, et al. (2014); Whitmee et al. (2015).

Os efeitos particulares da seca sobre as dimensões ambientais, econômicas e sociais, geram um conjunto de fatores de riscos que se sobrepõem (OPAS, 2015; CROSSMAN, 2018) e determinam os diversos impactos sobre a saúde e bem-estar humano (figura 2). Esses efeitos, muitas vezes repetidos, 
concatenados com os efeitos advindos dos outros processos de mudanças ambientais tendem a ampliar as ameaças sobre a saúde e o bem-estar humano. Para responder essa complexidade é necessário que haja uma política de seca com ênfase na gestão de redução de riscos. É importante estabelecer um conjunto de diretrizes operacionais direcionados a medidas proativas de preparação, mitigação e resposta (WMO; GWP, 2014). Em termos de saúde, ainda é necessário um sistema de vigilância e atenção que seja estruturado e integrado, com planos de preparação, monitoramento e alerta para redução de riscos e impactos em populações expostas à seca.

Figura 2 - Exemplos de fatores de risco associados à seca nas dimensões ambiental, econômica e social e seus impactos sobre a saúde



Fonte: Baseado em STANKE et al. (2013); SENA et al. (2014); OPAS (2015); YUSA et al. (2015); ALPINO et al. (2016); SENA et al. (2018).

Quando os riscos e impactos negativos de todos esses processos sobre a saúde humana são analisados, se instalam várias preocupações direcionadas ao setor saúde. Por exemplo: O setor saúde tem consciência dos impactos atuais e, se sim, quais são as preocupações para o presente e o futuro? A informação proveniente de setores externos, como exemplo, o setor meteorológico, gestão de águas, etc. Acarreta novas considerações quanto ao planejamento de ações? O setor está preparado tecnicamente e financeiramente para responder efetivamente aos possíveis efeitos negativos advindos de cada um desses processos? Que informação os gestores do setor da saúde necessitam para subsidiar as tomadas de 
decisão? Eles se apropriam do conhecimento e das evidências para a tomada de decisões mais efetivas, tanto para atuar no presente, quanto para planejar o futuro?

Responder a estas perguntas é importante porque em muitos países ainda se verifica uma certa apatia em relação aos impactos que essas mudanças influenciam no estado de saúde e bem-estar das populações. Mesmo em países que apresentam vulnerabilidades a sofrer severas consequências, ações proativas ainda são ausentes, insuficientes ou inadequadas (McMICHAEL et al., 2008; EBI, 2011).

\section{EVENTO CLIMÁTICO DE SECA NO BRASIL}

No Brasil, as médias anuais de precipitação também estão se alterando de forma extrema, principalmente em uma grande extensão de zonas semiáridas, abrangendo $1.128 .697 \mathrm{~km}^{2}$, onde a seca já é habitual (FUNCEME, 2017; BRASIL/MIN/SUDENE, 2018). Atualmente, essa área denominada de Semiárido Brasileiro abarca 1.262 municípios, concentrados em grande parte na região Nordeste, onde atinge nove estados, e na parte norte do estado de Minas Gerais. Essa região concentra mais de 27 milhões de brasileiros, em torno de $12,8 \%$ da população brasileira, em sua maior parte são pessoas pobres ou de baixa renda (BRASIL/MIN/SUDENE, 2018; BRASIL/MIN, 2019).

Além de seus diversos impactos, outras preocupações estão surgindo devido a possibilidade de a seca afetar permanentemente uma região, levando a um processo de desertificação (UNISDR, 2011; UNCCD, 2014; MARENGO et al., 2017). As mudanças climáticas em curso podem afetar o regime de chuvas e agravar esta tendência de desertificação e inviabilização da produção agrícola. Esses processos são associados a diferentes fatores de risco, como por exemplo, degradação da terra e dos recursos hídricos causadas por práticas agrícolas inapropriadas, principalmente em ecossistemas que já apresentam escassez de água (FAO, 2011), como é o caso do Semiárido Brasileiro (MAGALHÃES, 2016; SENA et al., 2018).

A depender de sua duração e intensidade, a seca se classifica em outras extensões com diferentes impactos sobre o ambiente e sobre a subsistência de uma sociedade (NDMA, 2019). De forma adicional aos impactos originados do processo de seca sobre o ambiente, os processos de desertificação e degradação da terra podem aumentar os danos sobre os demais recursos essenciais à vida, água potável, segurança alimentar, solo saudável, ar limpo, energia, minerais, desenvolvimento das plantas, e habitat dos animais (WILLIANS, 2014). Os impactos provenientes desses dois processos aumentam a possibilidade de desestabilizar a subsistência de populações que vivem em terras áridas ou semiáridas, podendo levar a uma crise de desenvolvimento humano invisível e ao processo de migração (UNCCD, 2014; VERNER, 2016). Como consequência, os danos nas dimensões econômicas e sociais (BUTLER et al., 2014; EBI; BOWEN, 2016) e na saúde e bem-estar das populações (STANKE et al., 2013; SENA et al., 2014; YUSA et al., 2015) tendem a ser ampliados. O quadro 1 apresenta as tipologias da seca com suas caracterizações e possíveis impactos sobre o ambiente e a sociedade. 
Quadro 1 - Tipos de seca e possíveis impactos sobre o ambiente e sociedade

\begin{tabular}{|c|c|c|}
\hline $\begin{array}{c}\text { Tipos } \\
\text { de seca }\end{array}$ & Caracterização & Impactos \\
\hline 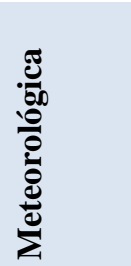 & $\begin{array}{l}\text { Atribui-se a variabilidade } \\
\text { climática natural com redução de } \\
\text { precipitação devido as condições } \\
\text { atmosféricas. } \\
\text { Varia entre as regiões, e em sua } \\
\text { durabilidade. }\end{array}$ & $\begin{array}{l}\text { Aumento da temperatura e de incidência solar. } \\
\text { Maior ocorrência de vento. } \\
\text { Diminui a umidade relativa do ar. } \\
\text { Aumenta a evaporação e transpiração. } \\
\text { Diminui a infiltração do solo e a recarga de águas superficiais. } \\
\text { Pode resultar em seca agrícola. }\end{array}$ \\
\hline 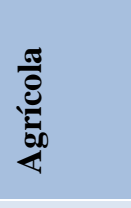 & $\begin{array}{l}\text { Depende das características da } \\
\text { seca meteorológica, com maior } \\
\text { foco na escassez de precipitação } \\
\text { e deficiência na umidade do solo. }\end{array}$ & $\begin{array}{l}\text { Estresse hídrico da terra, afetando os cultivos, podendo } \\
\text { impactar na germinação, crescimento e desenvolvimento (a } \\
\text { susceptibilidade da planta varia de acordo com suas } \\
\text { características biológicas e as propriedades físicas e biológicas } \\
\text { do solo). }\end{array}$ \\
\hline 焉 & $\begin{array}{l}\text { Deficiência no suprimento das } \\
\text { águas superficiais e subterrâneas. } \\
\text { Sua frequência e severidade são } \\
\text { definidas em uma escala de bacia } \\
\text { hidrográfica. }\end{array}$ & $\begin{array}{l}\text { Redução da vazão. } \\
\text { Afluência de reservatórios, lagos e lagoas. } \\
\text { Redução das zonas úmidas e do habitat de animais selvagens. } \\
\text { Os impactos sobre a dimensão econômica a mediano e longo } \\
\text { prazo. }\end{array}$ \\
\hline 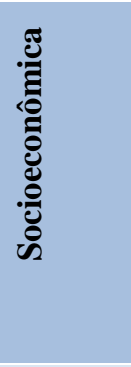 & $\begin{array}{l}\text { Ocorre quando a demanda por } \\
\text { algum tipo de bem econômico } \\
\text { (ex. água, alimento, energia) } \\
\text { ultrapassa a oferta, resultando em } \\
\text { determinantes sociais e } \\
\text { econômicos. }\end{array}$ & $\begin{array}{l}\text { Os impactos são decorrentes dos efeitos provenientes de todos } \\
\text { os tipos de seca. } \\
\text { Escassez de água, que pode gerar vários outros tipos de } \\
\text { impactos (ex. escassez de alimentos, aumento do preço de água, } \\
\text { doenças). } \\
\text { Insegurança alimentar (ex. escassez, preços inacessíveis, } \\
\text { desnutrição). } \\
\text { Conflitos devido a competitividade por água, e ou alimentos. } \\
\text { Migração de populações. }\end{array}$ \\
\hline 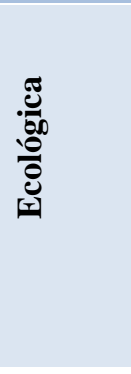 & $\begin{array}{l}\text { Quando o déficit no } \\
\text { abastecimento de água } \\
\text { naturalmente disponível se torna } \\
\text { prolongado e generalizado, } \\
\text { gerando estresses nos } \\
\text { ecossistemas. }\end{array}$ & $\begin{array}{l}\text { Aumento da temperatura. } \\
\text { Redução na absorção de carbono, aumentando os efeitos das } \\
\text { mudanças climáticas. } \\
\text { Redução da produtividade da biosfera e feedbacks do ciclo de } \\
\text { carbono, podendo influenciar em mais aquecimento. } \\
\text { Alteração nos regimes de umidade do ar e do solo. } \\
\text { Diminuição de produtividade do solo. } \\
\text { Intrusão de água salgada no sistema de água doce. } \\
\text { Migração de espécies. }\end{array}$ \\
\hline
\end{tabular}

Fonte: NDMA (2019) baseado em WILHITE e GLANTZ (1985); WILLIANS (2014).

Os riscos de sofrer severos impactos associados a eventos de seca depende da exposição dos sistemas de agricultura e de água, da vulnerabilidade local, dos setores, das populações e ecossistemas (SENA et al., 2017; MEZA et al., 2019). A capacidade de enfrentar ou de se adaptar às condições de seca também funcionam como mediadores dos impactos, assim como a duração do evento e suas complicações sobre o ambiente (IPCC, 2012; 2014; CDC, 2018).

Muitas vezes, as secas se estendem plurianualmente, isto é, perdem seu caráter puramente sazonal. No Brasil, a última seca plurianual ocorreu no semiárido entre o período 2012 a 2017 e causou grandes impactos sobre a região por abranger gradualmente os vários tipos de seca, como destacados no quadro 1 (MAGALHÃES, 2016; FUNCEME, 2017). Ultimamente, as secas nessa região têm se tornado mais severas gerando maiores prejuízos sobre a disponibilidade de água potável, a produção de alimentos e sobre a economia da região (BRASIL/CEMADEN/MCTI, 2015).

A agudização das secas implica em riscos cada vez maiores sobre a saúde das populações 
principalmente as mais vulnerabilizadas. Os grupos populacionais mais vulneráveis aos impactos negativos gerados pelas condições associadas à seca são: pessoas ou famílias com baixo ou insuficiente ingresso econômico, famílias que sobrevivem da produção de alimentos, pessoas que apresentam algum problema de saúde, idosos, mulheres gestantes, crianças, principalmente as menores de cinco anos de idade, pessoas que vivem em áreas remotas, incluindo populações indígenas, ou com dificuldade de acesso a recursos básicos como água, alimento e serviços de saúde (EBI, 2011; FLANAGAN et al., 2011; CARDONA et al., 2012; OPAS, 2015).

Embora a seca seja presente historicamente na região do Semiárido do Brasil, com ocorrência de fome, doenças, desnutrição e mortes que foram relatadas nos estudos de Alcântara Silva e colegas (2013) e Campos (2014), a busca do conhecimento para implantação de políticas públicas foi focada por muitos anos, no provimento de acesso à água, com a construção de açudes e para o desenvolvimento econômico e combate à pobreza. Depois, as preocupações se estenderam para a produção alimentar, incluindo a pecuária, e como amparar a população mais vulnerável, os sertanejos, que dependem da coleta, quando empregados, ou da venda dos alimentos para sobrevivência, para que não houvesse fome, desnutrição e morte (CAMPOS, 2014; MAGALHÃES, 2016). Vale ressaltar que em décadas mais recentes houve a implantação de programas em saúde para diminuir as mortes por doenças infecciosas (principalmente, diarreia) e desnutrição (VERNER, 2016). Porém, mesmo com os esforços na resposta em saúde, a preocupação em torno do conhecimento técnico com implementação de capacitações dos profissionais de saúde para diminuir os impactos sobre a saúde sempre foi escassa ou inexistente em alguns municípios. Mesmo hoje, esse conhecimento ainda é insuficiente, apesar das muitas evidências e da construção de guias orientados à gestão de redução de riscos associados à seca (OPAS, 2015; BRASIL/MS, 2015) no intuito de levar o conhecimento ao setor saúde.

\section{PERCEPÇÃO E RESPOSTA DO SETOR SAÚDE NO BRASIL}

Vários estudos apontam que a falta de conhecimento e de informação sobre os impactos que a seca pode causar sobre a saúde humana pode amplificar os efeitos negativos e a ocorrência de emergências em saúde pública (BRASIL/MS, 2015; OPAS, 2015; EBI e BOWEN, 2016; SENA et al., 2018).

Uma análise de indicadores chaves feita por alguns estudos utilizando os censos de 1991, 2000 e 2010 comparando os municípios da região do Semiárido do Brasil com os demais municípios do país mostra uma diferença significante. Apesar dos avanços em muitos indicadores em todas as regiões do país, a exemplos de educação, pobreza, renda, acesso á água encanada e melhorias na saúde humana com a diminuição na taxa de mortalidade infantil, a região do Semiárido Brasileiro ainda apresenta condições menos favoráveis em todos os indicadores analisados. Essas diferenças comprovam uma maior vulnerabilidade no Semiárido relacionada às dimensões social, ambiental e econômica (SENA et al., 2014; 2016; 2017). 
Ainda que os indicadores apresentem melhorias na região do Semiárido Brasileiro, e apesar dos guias construídos pela OPAS e MS, um estudo realizado por Sena e colegas (2018) aponta que ainda são ausentes ações de saúde para redução dos riscos associados às condições de seca nos municípios (pesquisados) dessa região. Nesse estudo (SENA et al., 2018) foi realizada uma análise de dados coletados por pesquisas qualitativas com 3 grupos, entre eles: as populações (por meio de organizações da sociedade civil), os gestores (em sua maioria de saúde) e os profissionais de saúde. O resultado apontou que a seca é percebida como um evento normal, que ocorre frequentemente na região, ocasionando falta de água e prejuízos na agricultura. Essa percepção reforça o reconhecimento da seca como um evento invisível no contexto da saúde pública, principalmente quando não se tem um planejamento de ações para redução de riscos que poderiam minimizar os impactos sobre a saúde humana. Por isso, é imprescindível a implementação de ações complementares e específicas de saúde, em seus diversos contextos.

Ainda em decorrência destas entrevistas, foram identificados diversos problemas de saúde que poderiam surgir ou se agravar em função da seca, como os problemas de saúde mental, desnutrição e deficiências nutricionais, doenças respiratórias, doenças transmitidas por vetores, neoplasias, doenças de transmissão hídrica, doenças relacionadas à falta de saneamento e higiene, assim como a intensificação de doenças devido o comprometimento e vulnerabilidade dos serviços de saúde e dos processos migratórios (SENA et al., 2018). A identificação desses problemas converge com os estudos em outras regiões e o conhecimento acumulado em pesquisas (Figura 2).

Com a projeção apontadas pelo PBMC e IPCC de que as áreas de seca globalmente, incluindo o Semiárido Brasileiro, tendem a piorar no futuro (PBMC, 2013; IPCC, 2014) é importante a elaboração, por parte dos municípios, de um plano integrado de ações proativas focadas na prevenção e redução de vulnerabilidades e riscos (DE NYS et al., 2016). O setor saúde deveria levar em consideração os vários aspectos e dimensões impactadas pela seca e as mudanças ambientais que agravam esse processo. Devido à multiplicidade de impactos negativos potenciais é fundamental uma revisão dos programas de atuação do governo e sociedade, com a finalidade de integrar ações setoriais.

Esta diversidade de dados provenientes de diversas fontes de informação constituem subsídios para o monitoramento de determinada situação e para a tomada de decisão dos gestores locais de saúde (BARCELLOS et al., 2016), principalmente considerando que existem, atualmente, tecnologias e dados que permitem a emissão de alertas para secas (EWBANK et al., 2019).

\section{RECOMENDAÇÕES PARA O SETOR SAÚDE}

Os efeitos negativos da seca sobre os determinantes sociais e ambientais e, a intensidade de futuros eventos devido às mudanças climáticas na região do Semiárido Brasileiro, combinados com as vulnerabilidades presentes, apresentam importantes desafios para o setor da saúde. O setor da saúde 
deve estar preparado para atuar em medidas preventivas para redução de riscos e vulnerabilidades que podem impactar sobre a saúde humana, considerando os efeitos a curto e longo prazo.

A capacidade de resposta governamental, incluindo o setor da saúde, e aqueles setores envolvidos no processo de um evento climático, assim como a maneira com que o município está organizado, também influenciam na magnitude dos múltiplos efeitos associados a um evento climático (WMO; GWP, 2014; EBI; BOWEN, 2016). Dessa forma, esse estudo recomenda algumas ações proativas para o setor da saúde, em quatro áreas chaves: evidência e informação, governança, trabalho em parcerias, e foco em capacitação, comunicação e conhecimento local.

\subsection{Evidência/informação}

As evidências mostram claramente a necessidade imediata de ações de prevenção relacionadas a interação ambiente e saúde (WHO, 2019). É imprescindível compreender as questões complexas em torno da seca, incluindo as características sociais, econômicas, ambientais, políticas e de saúde das populações, para assegurar um efetivo processo de gerenciamento de redução de risco de desastre associado à seca (WMO; GWP, 2014; OPAS, 2015; CROSSMAN, 2018). Ações de mitigação e de adaptação para mudanças climáticas também são necessárias nesse processo de gestão, uma vez que secas e escassez de água podem ser agravadas por essas mudanças (PATZ et al., 2012; IPCC, 2019; WHO, 2019).

Nesse contexto, é importante adotar um processo de formação baseada na capacidade crítica de avaliação, de análise e de síntese, tanto dos problemas e ameaças que afetam à população local, considerando a projeção de riscos futuros, quanto da capacidade de sua resposta a esses desafios (UNISDR, 2015; UNCCD, 2016; BANWELL et al., 2018). Os riscos para a saúde (apresentados na figura 2), desde os mais imediatos até os de longo prazo, devem ser incluídos no processo de avaliação de respostas e no planejamento de ações futuras de seca. Recomenda-se o registro oficial desses agravos nos sistemas de informação em saúde. Esses dados de informação permitiriam definir melhor o perfil epidemiológico das comunidades afetadas por seca e podem subsidiar um planejamento em saúde mais eficaz e eficiente.

\subsection{Governança}

Para atender as demandas de um planejamento integrado de ação em saúde voltado para a prevenção e redução de riscos e vulnerabilidades frente à seca, será necessário formular políticas públicas direcionadas aos problemas advindos da seca (VERNER, 2016). As diretrizes devem ser orientadas ao estabelecimento de parcerias com diversos setores e sociedade organizada, como meio de identificar os grupos populacionais mais vulneráveis, e com isso direcionar os investimentos financeiros, inclusive para capacitação de recursos humanos e para o fortalecimento de tecnologias e de 
infraestrutura de atenção e vigilância em saúde. Nesta circunstância, se faz necessário o desenvolvimento de um sistema de informação de doenças e óbitos no período agravados pela seca, a fim de construir uma base de dados que possa fortalecer as ações do setor da saúde para essa população. Outro sistema que seja integrado com o monitoramento e o alerta precoce de seca, considerando a duração e o agravamento que esse evento possa trazer. Por último, um sistema de informação em saúde e a criação de uma rede de segurança de respostas e emergências em saúde pública, com o estabelecimento ou aprimoramento de uma coordenação entre os setores e níveis de governo (JANCLOES et al., 2014; WMO; GWP, 2014; BARCELLOS et al., 2016).

A participação ativa do setor de saúde nas decisões políticas, em todos os níveis do governo, Municipal, Estadual e Federal, principalmente frente aos determinantes socioambientais locais é essencial para o planejamento de ações proativas. As comunidades também devem estar inseridas no processo de decisão, em particular, na organização e infraestrutura das políticas locais.

\subsection{Parcerias}

A colaboração e parceria de áreas afins para potencializar a informação e gerenciamento de ações integradas em saúde nas regiões acometidas pela seca e desertificação são essenciais, agindo com o intuito de garantir uma melhor preparação e resposta do setor saúde. Esse mecanismo deve considerar principalmente as agendas de mudanças climáticas, desenvolvimento sustentável e gestão de redução de riscos associados à seca (JANCLOES et al., 2014; OPAS, 2015). Ações intersetoriais podem ser planejadas entre setores e/ou programas chaves, como: agricultura, pecuária, meteorologia, gerenciamento de recursos hídricos, desenvolvimento regional, redução de risco de desastres saúde, meio ambiente, segurança alimentar, defesa civil, educação, desenvolvimento social, entre outros (SENA et al., 2014; OPAS, 2015; CROSSMAN, 2018). Questões menos pesquisadas ou focadas como por exemplos, diferença de gênero, doenças crônicas não transmissíveis, doenças de saúde mental, migração, permanente ou temporária, inclusão social e desigualdades sociais também devem ser discutidas para entrar no processo de tomada de decisões.

\subsection{Comunicação, capacitação e conhecimento local}

Agir em medidas de prevenção e redução de riscos e vulnerabilidades locais contribui para a redução de impactos sobre a saúde, a promoção de saúde e a melhoria sobre as condições e qualidade de vida das pessoas (EBI; BOWEN, 2016). Portanto, faz-se necessário promover conhecimento sobre os riscos das secas para a saúde e desenvolvimento humano e sobre as vulnerabilidades sociais e ambientais das diferentes áreas e comunidades que podem amplificar esses riscos (SENA et al., 2017).

O setor saúde também tem um papel fundamental no que diz respeito às comunidades afetadas localmente, pois determinados grupos populacionais e territórios podem se apresentar mais vulneráveis 
(MAGALHÃES, 2016). A implementação de medidas de adaptação e capacitação e a construção de planos de ações com a participação comunitária é essencial para aumentar a resiliência, tanto das comunidades locais, como dos serviços de saúde do próprio governo local (BOWEN et al., 2012; SENA et al., 2014). Faz-se importante na construção desse processo que as diferenças e experiências de cada comunidade sejam reconhecidas e respeitadas (FREITAS et al., 2014). É imprescindível estabelecer medidas educativas para construção do conhecimento com e para as comunidades em riscos (MARTINS et al., 2018), atribuindo o processo individual de construção pessoal mediante as necessidades e condições de cada um (WERNECK, 2008). Portanto, desenvolver estratégias de comunicação, educação e capacitação continuada para os profissionais de saúde e populações sobre a gestão de redução de riscos associados à seca, envolvendo ações de prevenção, promoção, preparação, alerta, resposta, recuperação e adaptação é indispensável para a construção do conhecimento em seca e saúde para o fortalecer de uma infraestrutura governamental e desenvolvimento humano na região.

\section{CONCLUSÃO}

Os efeitos da seca sobre a saúde pública são inúmeros e dependem da gravidade e duração do evento, das vulnerabilidades existentes e da infraestrutura de saúde local. A projeção de secas mais severas e frequentes podem ampliar esses efeitos, principalmente se não houver recursos disponíveis para mitigar os impactos. Em síntese, a situação para o futuro é mais preocupante do que se parece e o setor da saúde não se vê preparado para enfrentar os múltiplos e graves impactos negativos provenientes da seca, nem para responder a urgência do problema.

Prover o conhecimento dos riscos advindos das secas e identificar os elementos e grupos populacionais expostos e vulneráveis aos seus impactos oferece subsídios para a tomada de decisões no setor de saúde local. Para isso, é importante que o setor de saúde foque no processo completo e integrado de gestão de redução de riscos associados à seca, com ações de prevenção, preparação, resposta e recuperação, considerando a participação comunitária. As intervenções de avaliação e monitoramento também são indispensáveis para assegurar que lições aprendidas de eventos anteriores sejam implementadas em planos de adaptação futuros.

Portanto, a construção de resiliência, o aumento da capacidade adaptativa do setor saúde e das comunidades em riscos e o desempenho de ações proativas para redução dos impactos da seca sobre a saúde pública dependem do conhecimento e da informação para a tomada de decisões. 


\section{REFERENCIAS}

ALCÂNTARA SILVA, V. M. et al. O Desastre Seca no Nordeste Brasileiro. Polêmica, Rio de Janeiro, v.12, n.2, abr./jun., 2013. Disponível em:

http://www.epublicacoes.uerj.br/index.php/polemica/article/view/6431/4857

ALPINO, T. A.; SENA, A.M., FREITAS, C. M. Desastres relacionados à seca e saúde coletiva - uma revisão da literatura científica. Ciência \& Saúde Coletiva, Rio de Janeiro, v.21, n.3, p.809-820, 2016. doi: 10.1590/1413-81232015213.21392015.

BANWELL N.; RUTHERFORD, S.; MACKEY, B., Chu, C. Towards Improved Linkage of Disaster Risk Reduction and Climate Change Adaptation in Health: A Review. Int. J.

Environ. Res. Public Health, [s.1.], v.15, n.4, p.2-18, abr. 2018. doi:10.3390/ijerph15040793

BARCELLOS, C. et al. Mudanças climáticas e ambientais e as doenças infecciosas: cenários e incertezas para o Brasil. Epidemiol. Serv. Saúde, Brasília, v.18, n.3, p.215-304, jul./set. 2009.

BARCELLOS, C.; ROUX, E.; CECCATO, P. et al. An observatory to gather and disseminate information on the health-related effects of environmental and climate change. Rev Panam Salud Publica, [s.1.], v.40, n3, p.167-73, 2016.

BOWEN, K.J.; FRIEL, S.; EBI, K.; BUTLER, C.D.; FIONA MILLER, F.; MCMICHAEL, A.J. Governing for a healthy population: Towards an understanding of how decision-making will determine our global health in a changing climate. Int. J. Environ. Res. Public Health, [s.1.], v.9, n.1, p.55-72, 2012. doi:10.3390/ijerph9010055.

BRASIL. Centro Nacional de Monitoramento e Alertas de Desastres Naturais no Brasil. Relatório da Situação Atual da Seca no Semiárido e Impactos: Ano hidrológico 20142015. Brasília: Ministério da Ciência, Tecnologia e Informação, CEMADEN/MCTI, 2015. Disponível em: http://www.cemaden.gov.br/secaeimpactos/boletim-mensal-monitoramentoimpactos-seca-setembro-2015.php

BRASIL. Governo amplia semiárido para apoiar mais municípios que sofrem com seca. Ministério da Integração Nacional, 24 nov. 2017. Disponível em:

http://www.integracao.gov.br/ultimas-noticias/7047-governo-amplia-semiarido-para-apoiarmais-municipios-que-sofrem-com-seca. Acessado em: 25 jul. 2019.

BRASIL. Nova delimitação Semiárido. Ministério da Integração Nacional, nov. 2017. Disponível em:

http://www.sudene.gov.br/images/arquivos/semiarido/arquivos/Rela\%C3\%A7\%C3\%A3o_de _Munic\%C3\%ADpios_Semi\%C3\%A1rido.pdf

BUTLER, Colin. Climate change and global health: a new conceptual framework - Mini Review. CAB Reviews, [s.1.], v.9, n.27, 2014. Disponível em: doi: 10.1079/PAVSNNR20149027

CAMPOS, José Nilson B. Secas e políticas públicas no semiárido: ideias, pensadores e períodos. Estud. av., São Paulo, v. 28, n. 82, p. 65-88, dez. 2014. Disponível em: http://dx.doi.org/10.1590/S0103-40142014000300005 
CARDONA, O.D. et al. 2012: Determinants of risk: exposure and vulnerability. In: FIELD, C.B. et al. (eds.). Managing the Risks of Extreme Events and Disasters to Advance Climate Change Adaptation: A Special Report of Working Groups I and II of the Intergovernmental Panel on Climate Change (IPCC). Cambridge University Press, Cambridge: New York, 2012, p. 65-108.

CARVALHO, Otamar. As secas e seus impactos. In: MAGALHÃES, A. R. (coord.). A Questão da Água no Nordeste: Centro de Gestão de Estudos Estratégicos. Agência Nacional de Águas. Brasília: CGEE, 2012. p. 45-99.

CDC. Centers for Disease Control and Prevention. When every drop counts: Protecting public health during drought conditions: a guide for public health professionals. Atlanta: U.S. Department of Health and Human Services, 2010. Disponível em: https://www.cdc.gov/nceh/ehs/Docs/When_Every_Drop_Counts.pdf

CDC. Centers for Disease Control and Prevention. Preparing for the health effects of drought: a resource guide for public health professionals. 2018. Disponível em: https://www.cdc.gov/nceh/hsb/cwh/docs/CDC_Drought_Resource_Guide-508.pdf

CORVALAN, C.; Hales, S.; McMichael, A. Millennium Ecosystem Assessment.

Ecosystems and Human Well-Being: Health Synthesis. World Health Organization: Geneva, Switzerland, 2005. Disponível em:

www.millenniumassessment.org/documents/document.357.aspx.pdf

CROSSMAN, Neville D. Drought Resilience, Adaptation and Management Policy (DRAMP) Framework. Australia: UNCCD, jul. 2018. Disponível em:

https://www.unccd.int/sites/default/files/relevant-links/2018-

08/DRAMP_Policy_Framework.pdf

DAMANIA, R. et al. Uncharted Waters: The New Economics of Water Scarcity and Variability. Washington, DC: World Bank, 2017. doi:10.1596/978-1- 4648-1179-1.

DE NYS, E.; ENGLE, N.; QUINTANA, C.M. Marco e poder de mobilização. Em De Nys, E.; Engle, N.L.; Magalhães, A.R. (orgs.). Secas no Brasil: política e gestão proativas. Brasília, DF: Centro de Gestão e Estudos Estratégicos- CGEE; Banco Mundial, 2016. 292 p.

Disponível em: https://www.cgee.org.br/documents/10195/734063/seca_brasil-web.pdf

EBI, Kristie L. Resilience to the Health Risks of Extreme Weather Events in a Changing Climate in the United States. Int. J. Environ. Res. Public Health, [s.1.], v.8, p. 4582-4595, 2011. doi: 10.3390/ijerph8124582.

EBI, Kristie.; BOWEN, Kathryn. Extreme events as sources of health vulnerability: Drought as an example. Weather and Climate Extremes, [s.1.], v.1, n,11, p. 95- 102, mar. 2016. Disponível em: https://www.sciencedirect.com/science/article/pii/S221209471530030X

EWBANK, R.; PEREZ, C.; CORNISH, H.; WORKU, M.; WOLDETSADIK, S. Building resilience to El Niño-related drought: experiences in early warning and early action from Nicaragua and Ethiopia. Disasters, [s.1.], v.43, p.S345-S367, 2019. doi:10.1111/disa.12340. 
FAO. The state of the world's land and water resources for food and agriculture (SOLAW): Managing systems at risk. Rome: Food and Agriculture Organization of the United Nations, 2011.

FAO. Fao statistical yearbook 2013 world food and agriculture. Rome: Food and Agriculture Organization of the United Nations, p. 307, 2013. Disponível em: http://www.fao.org/3/i3107e/i3107e.pdf

FAO. Towards a water and food secure future: Critical Perspectives for Policy-makers. Food and Agriculture Organization of the United Nations: World Water Council, 2015. Disponível em: http://www.fao.org/3/a-i4560e.pdf

FIELD, C.B. et al. (eds.). Managing the Risks of Extreme Events and Disasters to Advance Climate Change Adaptation: A Special Report of Working Groups I and II of the Intergovernmental Panel on Climate Change (IPCC). Cambridge University Press, Cambridge: New York, 2012, 582p.

FIELD, C.B.; BARROS, V.R.; DOKKEN, D.J.; et al. (eds.) Climate Change 2014: Impacts, adaptation, and vulnerability. Part A: global and sectoral aspects (IPCC). Cambridge, UK/New York, USA: Cambridge University Press, 2014, 1132.

FLANAGAN, B. E.; GREGORY, E. W.; HALLISEY, E. J.; HEITGERD, J. L.; LEWIS, B. A Social Vulnerability Índex for Disaster Management. Journal of Homeland Security and Emergency Management, [s.1.], v. 8, n. 1, 2011.

FREITAS, C. M. et al. Desastres naturais e saúde: uma análise da situação do Brasil. Ciência \& Saúde Coletiva, [s.1.], v.19, n.9, p.3645-3656, 2014.

FUNCEME. FUNDAÇÃO CEARENSE DE METEOROLOGIA E RECURSOS HÍDRICOS. Secretaria dos Recursos Hídricos. Governo do Estado do Ceará. Brasil, 2017. Disponível em: www.funceme.br.

HAGENLOCHER, Michael; MEZA, I.; ANDERSON, C.C.; MIN, A. Drought vulnerability and risk assessments: state of the art, persistent gaps, and research agenda. Environ. Res. Lett, [s.1.], v.14, 2019. Disponível em: https://doi.org/10.1088/1748-9326/ab225d

JANCLOES, M.; THOMSON, M.; MÁÑEZ COSTA, M.; HEWITT, C.; CORVALAN, C.; et al. Climate services to improve public health. Int. J. Environ. Res. Public Health, [s.1.], v.11, p. 4555-4559, 2014. doi:10.3390/ijerph110504555.

LIMA R.C.C.; CAVALCANTE, A.M.B; PEREZ-MARIN, A.M. Desertificação e mudanças climáticas no semiárido brasileiro. Campina Grande: INSA- Instituto Nacional do Semiárido, 2011, 209p. Disponível em: https://portal.insa.gov.br/images/acervolivros/Desertifica\%C3\%A7\%C3\%A3o\%20e\%20Mudan\%C3\%A7as\%20Clim\%C3\%A1ticas $\% 20$ no\%20Semi\%C3\%A1rido\%20Brasileiro.pdf

MAGALHÃES, Antônio. Vida e seca no Brasil. In: DE NYS, E.; ENGLE, N.L.; MAGALHÃES, A.R. (orgs.). Secas no Brasil: política e gestão proativas. Brasília, DF: Centro de Gestão e Estudos Estratégicos- CGEE; Banco Mundial, 2016. 292 p. Disponível em: https://www.cgee.org.br/documents/10195/734063/seca_brasil-web.pdf 
MARENGO, J. A.; TORRES, R.R.; ALVES, L.M. Drought in Northeast Brazil: past, present and future. Theor Appl. Climatol, Springer, v.v129, n. 3-4, p. 1189-1200, ago. 2017. doi:10.1007/s00704-016-1840-8

MARTINS, E.D.; MOURA, A.A.; BERNARDO, A.A. O processo de construção do conhecimento e os desafios do ensino-aprendizagem. RPGE- Revista online de Política e Gestão Educacional, Araraquara, São Paulo, v.22, n.1, p. 410-423, jan./abr, 2018. DOI: 10.22633/rpge.v22.n.1.2018.10731.

McMICHAEL, A.J.; FRIEL, S.; NYONG, A.; CORVALAN, C. Global environmental change and health: impacts, enequalities, and the health sector. BMJ, [s.1.], v. 336, n.7637, p. 191194, 2008. doi: https://doi.org/10.1136/bmj.39392.473727.AD

MEZA, I.; HAGENLOCHER, M.; NAUMANN, G.; VOGT, J.; FRISCHEN, J. Drought vulnerability indicators for global-scale drought risk assessments. Publications Office of the European Union, Luxembourg, 2019. doi:10.2760/73844, JRC117546.

MYERS, S.S.; PATZ, J.A. Emerging Threats to Human Health from Global Environmental Change. Annu. Rev. Environ. Resour. [s.1.], v. 34, p.223-252, nov. 2009.

NDMC - National Drought Mitigation Center. Types of drought. University of Nebraska. School of Natural Resources. 2019. Disponível em:

https://drought.unl.edu/Education/DroughtIn-depth/TypesofDrought.aspx

DE SENA, A. R. M.; SILVA, E.L.; CORVALAN, C. Atuação do setor saúde frente a situações de seca. OPAS - Organização Pan-Americana de Saúde/ OMS-Organização Mundial da Saúde. Fundação Oswaldo Cruz/Ministério da Saúde, 2015. (série esenvolvimento Sustentável e saúde, 3). http://iris.paho.org/xmlui/handle/123456789/18468

PATZ, J.; CORVALAN, C.; HORTWITZ, P.; CAMPBELL-LENDRUM, D. Our Planet, Our Health, Our Future: Human Health and the Rio Conventions: biological diversity, climate change and desertification. Geneve: World Health Organization; 2012.

PBMC. Painel Brasileiro de Mudanças Climáticas. Contribuição do Grupo de Trabalho 1 ao Primeiro Relatório de Avaliação Nacional do Painel Brasileiro de Mudanças Climáticas. Sumário Executivo GT1. Rio de Janeiro: PBMC; 2013.

SENA, A. et al. Managing the Health Impacts of Drought in Brazil. Int. J. Environ. Res. Public Health, [s.1.], v.11, n.10, p.10737-10751, out. 2014. DOI:10.3390 / ijerph111010737

SENA, A.; FERNANDES, C.; LIMA E SILVA, E. (orgs.). Plano de Contingência para Emergência de Saúde Pública por Seca e Estiagem. Brasília: Secretaria de Vigilância em Saúde. Ministério da Saúde, 2015. 40p.: il. Disponível em: http://bvsms.saude.gov.br/bvs/publicacoes/plano_contingencia_emergencia_seca_estiagem.pd $\mathrm{f}$

SENA, A.; FREITAS, C.M.; BARCELLOS, C.; RAMALHO, W., Corvalan, C. Measuring the invisible: Analysis of the Sustainable Development Goals in relation to populations exposed to drought. Ciência Saúde Coletiva, [s.1.], v.21, n.3, p.671-84, mar. 2016. 
SENA, A. et al. Indicators to measure risk of disaster associated with drought: implications for the health sector. PLoS ONE, [s.1.], v.12, n.7, p. e0181394, 2017.

SENA, A.; FREITAS, C.; FEITOSA SOUZA, P.; CARNEIRO, F.; ALPINO, T. et al. Drought in the Semiarid Region of Brazil: Exposure, Vulnerabilities and Health Impacts from the Perspectives of Local Actors. PLOS Currents Disasters, [s.1.], out. 2018. doi: 10.1371/currents.dis.c226851ebd64290e619a4d1ed79c8639.

STANKE, C.; KERAC, M.; PRUDHOMME, C.; MEDLOCK, J.; MURRAY, V. Health Effects of Drought: a Systematic Review of the Evidence. PLOS Currents Disasters [s.1.], n. 1, jun. 2013. Disponível em: http://www.ncbi.nlm.nih.gov/pmc/articles/PMC3682759/.

UNEP. Global Environment Outlook 5. Environment for the future we want. Nairobi: United Nations Environment Programme, 2012. Disponível em: http://www.unep. org/geo/pdfs/geo5/GEO5_report_full_en.pdf

UNCCD. United Nations Convention to Combat Desertification. Desertification: A visual synthesis. França: UNCCD, 2011. Disponível em:

http://archive.zoinet.org/web/sites/default/files/publications/Desertification-SP.pdf

UNCCD. United Nations Convention to Combat Desertification. Desertification: the invisible frontline. 2. ed. França: UNCCD, 2014. ISBN: 9978-92-95043-74-9

UNCCD. United Nations Convention to Combat Desertification. The ripple effect: A Fresh Approach to Reducing Drought Impacts and Building Resilience. França: UNCCD, 2016. Disponível em: https://unccd. int/sites/default/files/documents/27072016_The ripple effect_ENG.pdf

UNISDR. Global Assessment Report on Disaster Risk Reduction: Revealing Risk, Redefining Development. International Strategy for Disaster Risk Reduction; Information Press: Oxford, UK, 2011; p. 54-69. Disponível em: www.preventionweb.net/english/hyogo/gar/2011/en/bgdocs/ GAR2011/GAR2011_Report_Chapter3.pdf

UNISDR. United Nations. International Strategy for Disaster Reduction. Sendai Framework for Disaster Risk Reduction 2015-2030. United Nations, 2015. Disponível em: https://www.preventionweb.net/files/43291_sendaiframeworkfordrren.pdf

VERNER Dorte. Vozes do povo: impactos socioeconômicos da seca no Nordeste do Brasil. In: DE NYS, E.; ENGLE, N.L.; MAGALHÃES, A.R. (orgs.). Secas no Brasil: política e gestão proativas. Brasília, DF: Centro de Gestão e Estudos Estratégicos- CGEE; Banco Mundial, 2016. 292 p. Disponível em: https://www.cgee.org.br/documents/10195/734063/seca_brasil-web.pdf

WERNECK, Vera Rudge. Sobre o processo de construção do conhecimento: o papel do ensino e da pesquisa. Ensaio: aval. pol. públ. Educ. [s.1.], v.14, n.51, p.173-196, 2006. http://dx.doi.org/10.1590/S0104-40362006000200003.

WHITMEE, S.; HAINES, A.; BEYRER, C.; BOLTZ, F.; CAPON, A.G.; SOUZA, B.F.S, et al. Safeguarding human health in the Anthropocene epoch: report of the Rockfeller 
Foundation-Lancet Commission on planetary health. The Lancet, [s.1.], v.386, n.10007, p.1973-2028, nov. 2015. https://doi.org/10.1016/S0140-6736(15)60901-1

WHO - World Health Organization. Healthy environments: Why do they matter, and what can we do? Geneva: World Health Organization, 2019 (WHO/CED/PHE/DO/19.01).

WILHITE, D.A.; GLANTZ, M.H. Understanding the Drought Phenomenon: The Role of Definitions. Water International, [s.1.], v.10, n.3, p. 111-120, 1985.

WILLIAMS, Christopher Alan. 2014. Heat and drought extremes likely to stress ecosystem productivity equally or more in a warmer, $\mathrm{CO} 2$ rich future. Perspective: Environ. Res. Lett. [s. 1.], v.9, 2014. doi:10.1088/1748-9326/9/10/101002.

WMO-World Meteorological Organization; Global Water Partnership; National Drought Mitigation Center. National Drought Management Policy Guidelines: A Template for Action. Integrated Drought Management Programme. Geneva: WMO, 2014.

YUSA, A.; BERRY, P.; CHENG, J.; OGDEN, N.; BONSAL, B.; STEWART, R., et al. Climate Change, Drought and Human Health in Canada. Int J Environ Res Public Health, [s.1.], v.12, n.7, p.8359-412, jul. 2015. 